\title{
Comparison of Artificial Compressibility Methods
}

\author{
Cetin Kiris ${ }^{1}$, Jeffrey Housman ${ }^{2}$, and Dochan Kwak ${ }^{3}$ \\ 1 NASA Ames Research Center, Moffet Field, CA, ckiris@mail arc.nasa.gov \\ 2 UC Davis, Davis, CA, jhousmanomath.ucdavis.edu \\ 3 NASA Ames Research Center, Moffet Field, CA, dkwakomail arc.nasa.gov
}

\begin{abstract}
Various artificial compressibility methods for calculating the three-dimensional incompressible Navier-Stokes equations are compared. Each method is described and numerical solutions to test problems are conducted. A comparison based on convergence behavior, accuracy, and robustness is given.
\end{abstract}

\section{Introduction}

The difficulty in computing solutions to the incompressible Navier-Stokes system of PDEs lies in satisfying the divergence-free velocity condition. Artificial compressibility methods, developed by A. Chorin [1], provide a mechanism to march in pseudo-time towards the divergence-free velocity field such that mass and momentum are conserved in the pseudo steady-state.

The classical artificial compressibility method transforms the mixed elliptic/parabolic type equations into a system of hyperbolic or parabolic equations in pseudo-time, which can be numerically integrated. The method has been generalized to curvilinear coordinates and used for various applications, Kiris et. al. [3].

Since the publication of Chorin's original paper many alternative forms of artificial compressibility have been developed. These methods include a generalized preconditioning matrix to equalize the wave speeds and the use of differential preconditioning, Turkel and Radespiel [7], as well as the addition of artificial viscosity such as an artificial Laplacian of pressure term in the continuity equation, Shen [6]. We present a direct comparison of four different versions of the artificial compressibility method on a series of test problems.

\section{Incompressible Equations and Artificial Compressibility}

The governing equations for incompressible, constant density and constant viscosity flow written in conservative form in generalized coordinates with the density absorbed by the non-dimensionalization of the pressure term are 


$$
I_{m} \frac{\partial \hat{Q}}{\partial t}+\frac{\partial\left(\hat{E}_{1}-\hat{E}_{1}^{v}\right)}{\partial \xi_{1}}+\frac{\partial\left(\hat{E}_{2}-\hat{E}_{2}^{v}\right)}{\partial \xi_{2}}+\frac{\partial\left(\hat{E}_{3}-\hat{E}_{3}^{v}\right)}{\partial \xi_{3}}=0
$$

where,

$$
\begin{aligned}
& I_{m}=\left[\begin{array}{llll}
0 & 0 & 0 & 0 \\
0 & 1 & 0 & 0 \\
0 & 0 & 1 & 0 \\
0 & 0 & 0 & 1
\end{array}\right] \hat{Q}=\frac{1}{J}\left[\begin{array}{c}
p \\
u \\
v \\
w
\end{array}\right] \hat{E}_{i}=\frac{1}{J}\left[\begin{array}{c}
U^{i} \\
u\left(U^{i}+\xi_{t}^{i}\right)+\xi_{x}^{i} p \\
v\left(U^{i}+\xi_{t}^{i}\right)+\xi_{y}^{i} p \\
w\left(U^{i}+\xi_{t}^{i}\right)+\xi_{z}^{i} p
\end{array}\right] \\
& \hat{E}_{i}^{v}=\frac{1}{R e J}\left[\begin{array}{c}
0 \\
\left(\nabla \xi^{i} \cdot \nabla \xi^{1}\right) u_{\xi_{1}}+\left(\nabla \xi^{i} \cdot \nabla \xi^{2}\right) u_{\xi_{2}}+\left(\nabla \xi^{i} \cdot \nabla \xi^{3}\right) u_{\xi_{3}} \\
\left(\nabla \xi^{i} \cdot \nabla \xi^{1}\right) v_{\xi_{1}}+\left(\nabla \xi^{i} \cdot \nabla \xi^{2}\right) v_{\xi_{2}}+\left(\nabla \xi^{i} \cdot \nabla \xi^{3}\right) v_{\xi_{3}} \\
\left(\nabla \xi^{i} \cdot \nabla \xi^{1}\right) w_{\xi_{1}}+\left(\nabla \xi^{i} \cdot \nabla \xi^{2}\right) w_{\xi_{2}}+\left(\nabla \xi^{i} \cdot \nabla \xi^{3}\right) w_{\xi_{3}}
\end{array}\right]
\end{aligned}
$$

Classical and Generalized Artificial Compressibility

The classical artificial compressibility formulation is derived by introducing an artificial compressibility relation in the continuity equation. This is achieved by adding a preconditioned pseudo-time derivative of the primitive variables $Q$ to equation 1 . The generalization of this approach is to begin with the conserved variables $W=(\rho, \rho u, \rho v, \rho w)^{T}$ and use the chain rule to derive the generalized preconditioned pseudo-time derivative. The classical preconditioning matrix, $\Gamma_{c}$, and the generalized preconditioning matrix, $\Gamma_{p}$, are

$$
\Gamma_{c}=\left[\begin{array}{cccc}
\frac{1}{\beta} & 0 & 0 & 0 \\
0 & 1 & 0 & 0 \\
0 & 0 & 1 & 0 \\
0 & 0 & 0 & 1
\end{array}\right], \quad \Gamma_{p}=\left[\begin{array}{cccc}
\frac{1}{\beta} & 0 & 0 & 0 \\
\frac{u}{\beta} & 1 & 0 & 0 \\
\frac{v}{\beta} & 0 & 1 & 0 \\
\frac{w}{\beta} & 0 & 0 & 1
\end{array}\right] \ldots
$$

where $\beta>0$ is the artificial compressibility parameter.

\section{Artificial Dissipation}

To assist in the dissipation of spurious pressure waves we add an artificial Laplacian of pressure term to the right-hand side of the classical artificial compressibility continuity equation. This term is scaled by a parameter $\epsilon$ and has the affect of adding a second difference artificial dissipation term to the continuity equation.

\section{Differential Preconditioning}

The artificial dissipation described above manipulates the physical dissipation properties of the PDE system. Alternatively we can manipulate the convective properties of the system. Following Turkel and Radespiel [7] a pseudo-time derivative of the Laplacian of pressure can be added to the continuity equation along with the standard pseudo-time derivative of pressure. This term is also scaled by $\epsilon$ and will have an effect of propagating the low-frequency components of error more quickly than the high-frequency components which will be dissipated by the discretization scheme. 


\section{Numerical Results}

The INS3D code $[4],[5],[3]$ has been adapted to include each of the artificial compressibility methods described. An implicit line symmetric Gauss-Seidel relaxation scheme is used with fully-implicit boundary conditions. Iterations are performed until the residual of the nonlinear system has been reduced nine orders of magnitude in the $l^{2}$ norm. Results for $\beta=1,10,100$ and $C F L=1$ and $C F L=1000$ are provided. For the artificial dissipation method $\epsilon$ values of $1.0^{-2}, 1.0^{-1}, 1.0^{+0}$ are used to scale the Laplacian term. The differential preconditioned method uses values of $\epsilon=1.0^{-1}, 1.0^{+0}, 1.0^{+1}$. For each testcase the inlet velocity is specified and a constant pressure is enforced at the outlet. $P=1,2,3,4$ denotes the version of the artificial compressibility method.

Test 1: Inviscid Flow in a Square Duct

Each method is used to calculate the inviscid flow in a square duct with dimensions $10 \times 1 \times 1$ non-dimensional units. The exact solution is $Q=$ $(0,1,0,0)^{T}$. A grid of dimension $33 \times 9 \times 9$ is used. Table 1 displays the number of iterations required. Each of the methods computed the correct solution up to double precision. For $\beta=1$ the generalized preconditioned method has the best convergence. For $\beta>1$, the classical has the best convergence rate with the exception of $C F L=1000$ where the differential preconditioning scheme is slightly better.

Table 1. Inviscid square duct: Number of iterations for residual reduction of 9 orders of magnitude in the discrete $L^{2}$ norm.

\begin{tabular}{|r|r|r|r|r|r|r|r|r|r|r|}
\hline & & $\mathrm{P}=1$ & $\mathrm{P}=2$ & & $\mathrm{P}=3$ & & & $\mathrm{P}=4$ & & \\
\hline $\mathrm{CFL}$ & $\beta$ & & & $\epsilon=$ & 0.01 & 0.10 & 1.00 & 0.10 & 1.00 & 10.0 \\
\hline \hline 1 & 1 & 220 & 209 & & 325 & 1876 & $>9000$ & 269 & 875 & 6992 \\
\hline & 10 & 151 & 160 & & 158 & 266 & 1874 & 165 & 309 & 2290 \\
\hline & 100 & 242 & 261 & & 243 & 250 & 328 & 246 & 291 & 1093 \\
\hline \hline 1000 & 1 & 212 & 202 & & 314 & 1867 & $>9000$ & 212 & 213 & 219 \\
\hline & 10 & 137 & 137 & & 145 & 255 & 1863 & 137 & 137 & 131 \\
\hline & 100 & 238 & 255 & & 239 & 242 & 304 & 238 & 238 & 238 \\
\hline
\end{tabular}

Test 2: Viscous Flow in a Circular Pipe

A simple viscous flow in a circular pipe of radius one and length ten is computed. A Reynolds number of 1000 is used for which an exact solution is derived. Grids of dimension $17 \times 9 \times 9,33 \times 17 \times 17$, and $65 \times 33 \times 33$ are used. Each method is verified to produce second order accurate results for $\beta=10$. Figure 1 plots the normalized $l^{2}$ residual for varying $\beta$ and $C F L=1000$. Table 2 displays the number of iterations required to converge on the finest mesh. The third and fourth methods fail to converge for low $C F L$ numbers and the artificial dissipation scheme is especially sensitive to the $\epsilon$ parameter. 
For high $C F L$ and high $\beta$ the differential preconditioning becomes effective, but a moderate $\beta$ must be used for accuracy purposes.
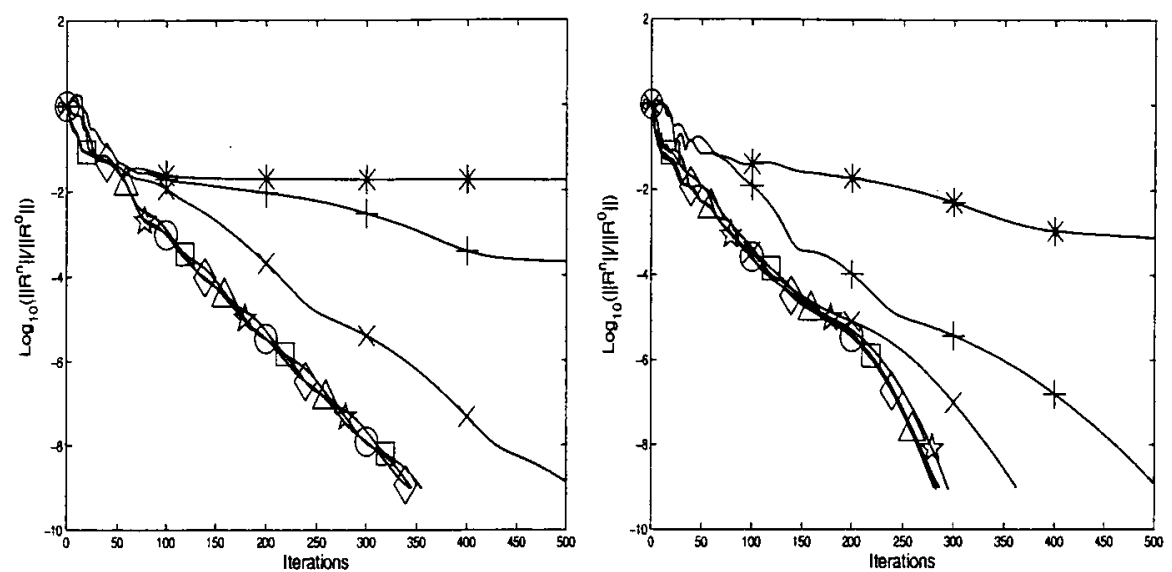

Fig. 1. Viscous Straight Pipe (Grid $65 \times 33 \times 33, R e=1000, C F L=1000$ ): Comparison of the convergence between preconditioning methods for $\beta=1$ (left) and $\beta=10$ (right). $\circ P=1 ; \square P=2 ; \times P=3, \epsilon=1.0^{-2} ;+P=3, \epsilon=1.0^{-1}$; * $P=3, \epsilon=1.0^{+0} ; \diamond P=4, \epsilon=1.0^{-1} ; \triangle P=4, \epsilon=1.0^{+0} ; \star P=4, \epsilon=1.0^{+1}$.

Table 2. Viscous Straight Pipe: Number of iterations for residual reduction of 9 orders of magnitude in the discrete $L^{2}$ norm.

\begin{tabular}{|r|r|r|r|r|l|l|l|l|l|l|}
\hline & & $\mathrm{P}=1$ & $\mathrm{P}=2$ & & $\mathrm{P}=3$ & & & $\overline{\mathrm{P}}=4$ & & \\
\hline $\mathrm{CFL}$ & $\beta$ & & & $\epsilon=$ & 0.01 & 0.10 & 1.00 & 0.10 & 1.00 & 10.0 \\
\hline \hline 1 & 1 & 352 & 363 & & 518 & $>2500$ & $>2500$ & 399 & 1233 & $>2500$ \\
\hline & 10 & 290 & 301 & & 364 & 500 & $>2500$ & 374 & 1577 & $>2500$ \\
\hline & 100 & 734 & 743 & & 753 & 1044 & 1242 & 922 & 1130 & $>2500$ \\
\hline 1000 & 1 & 343 & 355 & & 509 & $>2500$ & $>2500$ & 343 & 343 & 345 \\
\hline & 10 & 281 & 286 & & 362 & 503 & $>2500$ & 281 & 283 & 295 \\
\hline & 100 & 744 & 754 & & 748 & 1030 & 1210 & 743 & 736 & 688 \\
\hline
\end{tabular}

\section{Test 3: Viscous Flow in an L-shaped Duct}

A more complicated viscous flow in a square duct with a $90^{\circ}$ bend is used for the final test. The geometry used is described in Humphrey [2], where experimental results were obtained for Reynolds number 790 . A grid of dimension $65 \times 33 \times 33$ is used. Figure 2 plot the residual for varying $\beta$ and $C F L=1000$. Table 3 displays the number of iterations required. The symbol $* * * *$ denotes 
the method failed to converge.Figure 3 displays a comparison of the different computed solutions with the experimental data for $\beta=1,10,100$ at $\theta=90^{\circ}$ plane of the curved duct. Robustness is an issue for the third and fourth schemes. Comparing the experimental data with the computed solutions we observe that using $\beta>10$ leads to poor solution accuracy and $\beta=1$ is the most accurate.
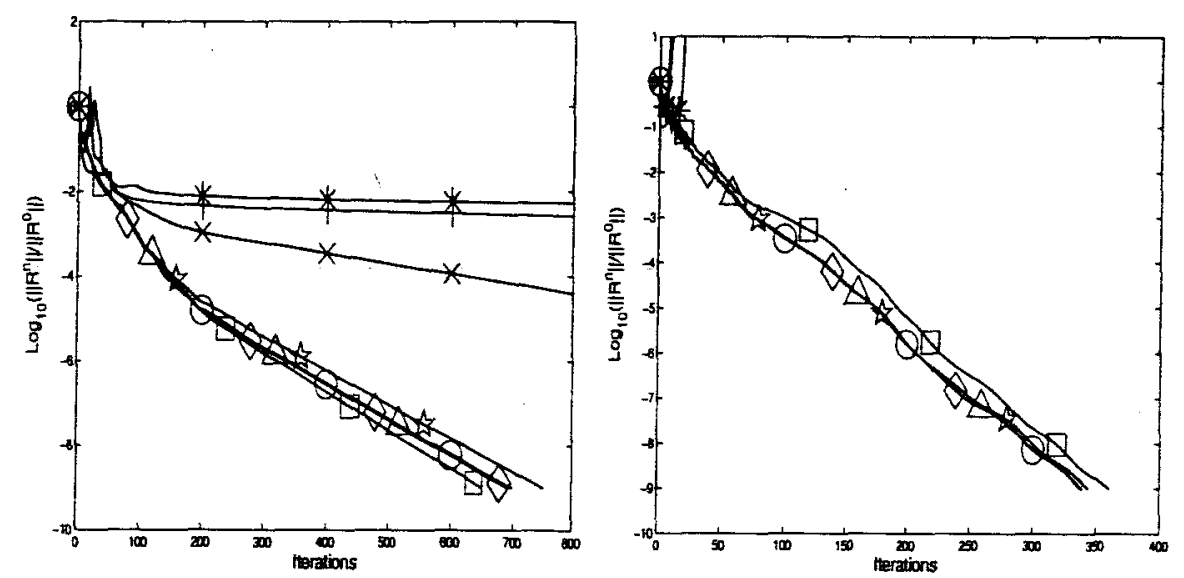

Fig. 2. Viscous Square Duct with $90^{\circ}$ bend (Grid $65 \times 33 \times 33, R e=790, C F L=$ 1000): Comparison of the convergence between preconditioning methods for $\beta=1$ (left) and $\beta=10$ (right). $\circ \quad P=1 ; \square \quad P=2 ; \times \quad P=3, \epsilon=1.0^{-2} ;+P=$ $3, \epsilon=1.0^{-1} ; * \quad P=3, \epsilon=1.0^{+0} ; \diamond P=4, \epsilon=1.0^{-1} ; \triangle P=4, \epsilon=1.0^{+0}$ $\star P=4, \epsilon=1.0^{+1}$.

\section{Conclusion}

Four variations of the artificial compressibility method have been implemented and compared on a series of test problems. The classical and generalized artificial compressibility methods have almost identical convergence rates on each test case for every combination of the $C F L$ and $\beta$ parameters. The artificial dissipation and differential preconditioning methods were not able to converge for all parameters on certain problems showing a lack of robustness for these methods. High values of $\beta$ lead to poor accuracy for all the methods considered. For moderate values of $1 \leq \beta \leq 10$ the classical and generalized versions appear to be the most accurate. These two versions will be evaluated for more complicated engineering applications. 
Table 3. Viscous Square Duct with $90^{\circ}$ bend: Number of iterations for residual reduction of 9 orders of magnitude in the discrete $L^{2}$ norm.

\begin{tabular}{|r|r|l|l|l|l|l|l|l|l|l|}
\hline & & $\mathrm{P}=1$ & $\mathrm{P}=2$ & & $\mathrm{P}=3$ & & & $\mathrm{P}=4$ & & \\
\hline $\mathrm{CFL}$ & $\beta$ & & & $\epsilon=$ & 0.01 & 0.10 & 1.00 & 0.10 & 1.00 & 10.0 \\
\hline \hline 1 & 1 & 697 & 655 & & 2764 & 5481 & 9999 & 1141 & $* * * *$ & $* * * *$ \\
\hline & 10 & 341 & 365 & & $* * * *$ & $* * * *$ & $* * * *$ & 388 & $* * * *$ & $* * * *$ \\
\hline & 100 & 524 & 594 & & $* * * *$ & $* * * *$ & $* * * *$ & $* * * *$ & $* * * *$ & $* * * *$ \\
\hline \hline 1000 & 1 & 693 & 653 & & 2760 & 5483 & 9999 & 693 & 699 & 750 \\
\hline & 10 & 338 & 361 & & $* * * *$ & $* * * *$ & $* * * *$ & 338 & 339 & 344 \\
\hline & 100 & 534 & $* * * *$ & & $* * * *$ & $* * * *$ & $* * * *$ & 534 & 535 & 543 \\
\hline
\end{tabular}
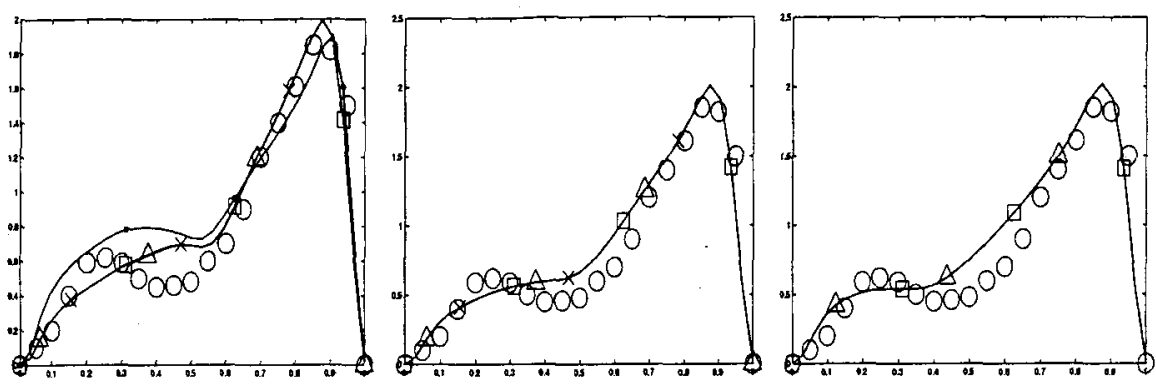

Fig. 3. Comparison of the solution at $\theta=90^{\circ}$ and $\beta=1$ (left), $\beta=10$ (center), and $\beta=100$ (right). $\circ$ Exper.; $\square P=1 ; \triangle P=2 ; \times P=3 ; \bullet P=4$.

\section{References}

1. A.J. Chorin. A Numerical Method for Solving Incompressible Viscous Flow Problems. J. Comput. Phys., 2(12), 1967.

2. J.A. Humphrey, A.M. Taylor, and J. Whitelaw. Laminar Flow in a Square Duct of Strong Curvature. J. Fluid Mech., 83(3):509-527, 1977.

3. C. Kiris, D. Kwak, and S. Rogers. Incompressible Navier-Stokes Solvers in Primitive Variables and Their Applications to Steady and Unsteady Flow. In M.M. Hafez, editor, Numerical Simulations of Incompressible Flows, pages 3-34. World Scientific Publishing, 2003.

4. D. Kwak, J.L.C. Chang, S.P. Shanks, and S.R. Chakravarthy. A ThreeDimensional Incompressible Navier-Stokes Solver Using Primitive Variables. AIAA J., 24(3), 1986.

5. S.E. Rogers, D. Kwak, and C. Kiris. Steady and Unsteady Solutions of the Incompressible iNavier-Stokes Equations. $A \dot{I} A \dot{A} J$., $\overline{2} \overline{9}(4), 19 \overline{9} 1$.

6. Jie Shen. Pseudo-Compressibility Methods for the Unsteady Incompressible Navier-Stokes Equations. In B. Guo, editor, Beijing Symposium on Nonlinear Evolution Equations and Infinite Dynamical Systems, 1994.

7. E. Turkel and R. Radespiel. Preconditioning Methods for Multidimensional Aerodynamics. In VKI Lecture Notes, 1997. 
Presentation mate

Comparison

of

Artificial Compressibility Methods

Cutin Kris

NASA Ames Research Center, Moffet Field, CA ckiris@mail.arc.nasa.gov

Jeffrey Housman

UC Davis, Davis, CA

jhousman@math.ucdavis.edu

Dochan Kwa

NASA Ames Research Center, Moffet Field, CA dkwak@mail.arc.nasa.gov

1 


\section{Abstract}

Various artificial compressibility methods for calculating the three-dimensional incompressible Navier-Stokes equations are compared. Each method is described and numerical solutions to test problems are conducted. A comparison based on convergence behavior, accuracy, and robustness is given. 
- Introduction

- Governing Equations

- Artificial Compressiblity Methods

- Classical Artificial Compressiblity

- Generalized Artificial Compressibility

- Artificial Dissipation

- Differential Preconditioning

- Numercial Examples

- Conclusion 


\section{Introduction}

The difficulty in computing solutions to the incompressible Navier-Stokes system of .PDEs lies in satisfying the divergence-free velocity condition. Artificial compressibility methods, developed by A. Chorin [1], provide a mechanism to march in pseudo-time towards the divergence-free velocity field such that mass and momentum are conserved in the pseudo steady-state.

The classical artificial compressibility method transforms the mixed elliptic/parabolic type equations into a system of hyperbolic or parabolic equations in pseudo-time, which can be numerically integrated. The method has been generalized to curvilinear coordinates and used for various applications, Kiris et. al. [2]. 
Since the publication of Chorin's original paper many alternative forms of artificial compressibility have been developed. These methods include a generalized preconditioning matrix to equalize the wave speeds and the use of differential preconditioning, Turkel and Radespiel [3], as well as the addition of artificial viscosity such as an artificial Laplacian of pressure term in the continuity equation, Shen [4]. We present a direct comparison of four different versions of the artificial compressibility method on a series of test problems. These problems include inviscid flow in a square duct, viscous flow in a circular pipe, and viscous flow in a square duct with strong curvature. 


\section{Governing Equations}

The governing equations for incompressible, constant density and constant viscosity flow written in conservative form in generalized coordinates with the density absorbed by the nondimensionalization of the pressure term are

$$
I_{m} \frac{\partial \widehat{Q}}{\partial t}+\frac{\partial\left(\hat{E}_{1}-\hat{E}_{1}^{v}\right)}{\partial \xi_{1}}+\frac{\partial\left(\hat{E}_{2}-\hat{E}_{2}^{v}\right)}{\partial \xi_{2}}+\frac{\partial\left(\hat{E}_{3}-\hat{E}_{3}^{v}\right)}{\partial \xi_{3}}=0,
$$

where,

$$
\begin{aligned}
& I_{m}=\left[\begin{array}{llll}
0 & 0 & 0 & 0 \\
0 & 1 & 0 & 0 \\
0 & 0 & 1 & 0 \\
0 & 0 & 0 & 1
\end{array}\right] \hat{Q}=\frac{1}{J}\left[\begin{array}{c}
p \\
u \\
v \\
w
\end{array}\right] \widehat{E}_{i}=\frac{1}{J}\left[\begin{array}{c}
U^{i} \\
u\left(U^{i}+\xi_{t}^{i}\right)+\xi_{x}^{i} p \\
v\left(U^{i}+\xi_{i}^{i}\right)+\xi_{i}^{i} p \\
w\left(U^{i}+\xi_{t}^{i}\right)+\xi_{z}^{z} p
\end{array}\right] \\
& \hat{E}_{i}^{v}=\frac{1}{R e J}\left[\begin{array}{c}
\left(\nabla \xi^{i} \cdot \nabla \xi^{1}\right) u_{\xi_{1}}+\left(\nabla \xi^{i} \cdot \nabla \xi^{2}\right) u_{\xi_{2}}+\left(\nabla \xi^{i} \cdot \nabla \xi^{3}\right) u_{\xi_{3}} \\
\left(\nabla \xi^{i} \cdot \nabla \xi^{1}\right) w_{\xi_{1}}+\left(\nabla \xi^{i} \cdot \nabla \xi^{2}\right) v_{\xi_{2}}+\left(\nabla \xi^{2} \cdot \nabla \xi^{3}\right) v_{\xi_{3}} \\
\left(\nabla \xi^{i} \cdot \nabla \xi^{1}\right) w_{\xi_{1}}+\left(\nabla \xi^{i} \cdot \nabla \xi^{2}\right) w_{\xi_{2}}+\left(\nabla \xi^{i} \cdot \nabla \xi^{3}\right) w_{\xi_{3}}
\end{array}\right] \\
& \frac{1}{J}=\frac{\partial x^{1}}{\partial \xi^{1}} \frac{\partial x^{2}}{\partial \xi^{2}} \frac{\partial x^{3}}{\partial \xi^{3}}+\frac{\partial x^{1}}{\partial \xi^{2}} \frac{\partial x^{2}}{\partial \xi^{3}} \frac{\partial x^{3}}{\partial \xi^{1}}+\frac{\partial x^{1}}{\partial \xi^{3}} \frac{\partial x^{2}}{\partial \xi^{1}} \frac{\partial x^{3}}{\partial \xi^{2}}- \\
& \frac{\partial x^{1}}{\partial \xi^{1}} \frac{\partial x^{2}}{\partial \xi^{3}} \frac{\partial x^{3}}{\partial \xi^{2}}-\frac{\partial x^{1}}{\partial \xi^{2}} \frac{\partial x^{2}}{\partial \xi^{1}} \frac{\partial x^{3}}{\partial \xi^{3}}-\frac{\partial x^{1}}{\partial \xi^{3}} \frac{\partial x^{2}}{\partial \xi^{2}} \frac{\partial x^{3}}{\partial \xi^{1}} \\
& U^{i}=\xi_{x}^{i} u+\xi_{y}^{i} v+\xi_{z}^{i} w \text {. }
\end{aligned}
$$




\section{Artificial Compressiblity Methods}

The artificial compressibility formulation is derived by introducing an artificial compressibility relation,

$$
\rho^{*}=\frac{p}{\beta},
$$

where $p$ is the pressure and $\beta>0$ is the artificial sound speed. Using this relation we may add a preconditioned pseudo-time derivative of the primitive variables $Q$ to equation 1 . Four forms of artificial compressiblity will be discussed.

- Classical Artificial Compressiblity

- Generalized Artificial Compressibility

- Artificial Dissipation

- Differential Preconditioning 


\section{Classical Artificial Compressiblity}

The classical artificial compressibility method uses equation 2 only in the continuity equation.

This leads to the following preconditioned system of equations,

$$
\Gamma_{c} \frac{\partial \widehat{Q}}{\partial \tau}+I_{m} \frac{\partial \widehat{Q}}{\partial t}+\frac{\partial\left(\hat{E}_{i}-\hat{E}_{i}^{v}\right)}{\partial \xi_{i}}=0 .
$$

The classical preconditioning matrix is given by,

$$
\Gamma_{c}=\left[\begin{array}{cccc}
\frac{1}{\beta} & 0 & 0 & 0 \\
0 & 1 & 0 & 0 \\
0 & 0 & 1 & 0 \\
0 & 0 & 0 & 1
\end{array}\right]
$$




\section{Generalized Artificial Compressiblity}

To generalize the above approach we start with the conserved variables $W=(\rho, \rho u, \rho v, \rho w)^{T}$. Using the chain rule and equation 2 we obtain,

$$
\frac{\partial W}{\partial \tau}=\frac{\partial W}{\partial Q} \frac{\partial Q}{\partial \tau}=\Gamma_{p} \frac{\partial Q}{\partial \tau},
$$

where

$$
\Gamma_{p}=\left[\begin{array}{cccc}
\frac{1}{\beta} & 0 & 0 & 0 \\
\frac{u}{\beta} & 1 & 0 & 0 \\
\frac{v}{\beta} & 0 & 1 & 0 \\
\frac{w}{\beta} & 0 & 0 & 1
\end{array}\right]
$$

Substituting $\Gamma_{p}$ for $\Gamma_{c}$ we obtain the generalized precondition system of equations,

$$
\Gamma_{p} \frac{\partial \widehat{Q}}{\partial \tau}+I_{m} \frac{\partial \widehat{Q}}{\partial t}+\frac{\partial\left(\hat{E}_{i}-\widehat{E}_{i}^{v}\right)}{\partial \xi_{i}}=0
$$




\section{Artificial Dissipation}

Introducing a finite sound speed into the incompressible equations creates artificial pressure waves which must be propagated out of the solution domain in pseudo-time.

Alternatively the artificial pressure waves can be dissipated by adding an artificial Laplacian of pressure to the modified continuity equation in equation 3 . Where the viscous fluxes $\widehat{E}_{i}^{v}$ are replaced by,

$$
\tilde{E}_{i}^{v}=\frac{1}{J}\left[\begin{array}{c}
\frac{\epsilon}{\beta}\left(\left(\nabla \xi^{i} \cdot \nabla \xi^{1}\right) p_{\xi_{1}}+\left(\nabla \xi^{i} \cdot \nabla \xi^{2}\right) p_{\xi_{2}}+\left(\nabla \xi^{i} \cdot \nabla \xi^{3}\right) p_{\xi_{3}}\right) \\
\frac{1}{R e}\left(\left(\nabla \xi^{i} \cdot \nabla \xi^{1}\right) u_{\xi_{1}}+\left(\nabla \xi^{i} \cdot \nabla \xi^{2}\right) u_{\xi_{2}}+\left(\nabla \xi^{i} \cdot \nabla \xi^{3}\right) u_{\xi_{3}}\right) \\
\frac{1}{R e}\left(\left(\nabla \xi^{i} \cdot \nabla \xi^{1}\right) v_{\xi_{1}}+\left(\nabla \xi^{i} \cdot \nabla \xi^{2}\right) v_{\xi_{2}}+\left(\nabla \xi^{i} \cdot \nabla \xi^{3}\right) v_{\xi_{3}}\right) \\
\frac{1}{R e}\left(\left(\nabla \xi^{i} \cdot \nabla \xi^{1}\right) w_{\xi_{1}}+\left(\nabla \xi^{i} \cdot \nabla \xi^{2}\right) w_{\xi_{2}}+\left(\nabla \xi^{i} \cdot \nabla \xi^{3}\right) w_{\xi_{3}}\right)
\end{array}\right] .
$$

To obtain,

$$
\Gamma_{c} \frac{\partial \widehat{Q}}{\partial \tau}+I_{m} \frac{\partial \widehat{Q}}{\partial t}+\frac{\partial\left(\widehat{E}_{i}-\widetilde{E}_{i}^{v}\right)}{\partial \xi_{i}}=0
$$




\section{Differential Preconditioning}

The classical artificial compressibility method uses a standard pseudo-time derivative to convect the artificial pressure waves out of the domain. So both high and low frequency errors are convected at constant wave speeds. Alternatively, the time derivative of the Laplacian of pressure term can be added to the first equation in the system. This forces the low frequecy errors to travel at faster speeds then there high frequency counterparts. The high frequency errors will be damped by the discretization and relaxation schemes.

This produces a system of the form,

$$
\Gamma_{c} \frac{\partial}{\partial \tau}\left[I-I_{\epsilon} \Delta\right] \widehat{Q}+I_{m} \frac{\partial \widehat{Q}}{\partial t}+\frac{\partial\left(\widehat{E}_{i}-\widehat{E}_{i}^{v}\right)}{\partial \xi_{i}}=0,
$$

where

$$
I_{\epsilon}=\left[\begin{array}{llll}
\epsilon & 0 & 0 & 0 \\
0 & 0 & 0 & 0 \\
0 & 0 & 0 & 0 \\
0 & 0 & 0 & 0
\end{array}\right], \Delta=\frac{\partial^{2}}{\partial \xi_{1}^{2}}+\frac{\partial^{2}}{\partial \xi_{2}^{2}}+\frac{\partial^{2}}{\partial \xi_{3}^{2}} .
$$




\section{Numerical Examples}

The INS3D code has been adapted to include each of the artificial compressibility methods described. An implicit line symmetric Gauss-Seidel relaxation scheme is used with fully-implicit boundary conditions. Iterations are performed until the residual has been reduced nine orders of magnitude in the $l^{2}$ norm. Results for $\beta=1,10,100$ and $C F L=1$ and $C F L=1000$ are provided. For the artificial dissipation method $\epsilon$ values of $1.0^{-2}$ and $1.0^{-1}$ are used to scale the Laplacian term. The differential preconditioned method uses values of $\epsilon=1.0^{-1}$ and $1.0^{+1}$. For each test-case the inlet velocity is specified and a constant pressure is enforced at the outlet.

- Inviscid Square Duct

- Viscous Circular Pipe

- Viscous Square Duct with Strong Curvature 


\section{Inviscid Square Duct}

Each method is used to calculate the inviscid flow in a square duct with dimensions $10 \times 1 \times 1$ non-dimensional units. The exact solution is $Q=(0,1,0,0)^{T}$. A grid of dimension $33 \times 9 \times 9$ is used. The table below displays the number of iterations required. Each of the methods computed the correct solution up to double precision. For $\beta=1$ the generalized preconditioned method has the best convergence. For $\beta>1$, the classical has the best convergence rate with the exception of $C F L=1000$ where the differential preconditioner scheme is slightly better. $P=1,2,3,4$ denotes the version of the artificial compressibility method.

\begin{tabular}{|c|c|c|c|c|c|c|c|c|}
\hline CFL & $\beta / \epsilon$ & $P=1$ & $P=2$ & & $\begin{array}{l}P=3 \\
0.01\end{array}$ & 0.10 & $\begin{array}{l}P=4 \\
0.10\end{array}$ & 10.0 \\
\hline \hline 1 & 1 & 220 & 209 & & 325 & 1876 & 269 & 6992 \\
\hline & 10 & 151 & 160 & & 158 & 266 & 165 & 2290 \\
\hline & 100 & 242 & 261 & & 243 & 250 & 246 & 1093 \\
\hline 1000 & 1 & 212 & 202 & & 314 & 1867 & 212 & 219 \\
\hline & 10 & 137 & 137 & & 145 & 255 & 137 & 131 \\
\hline & 100 & 238 & 255 & & 239 & 242 & 238 & 238 \\
\hline
\end{tabular}




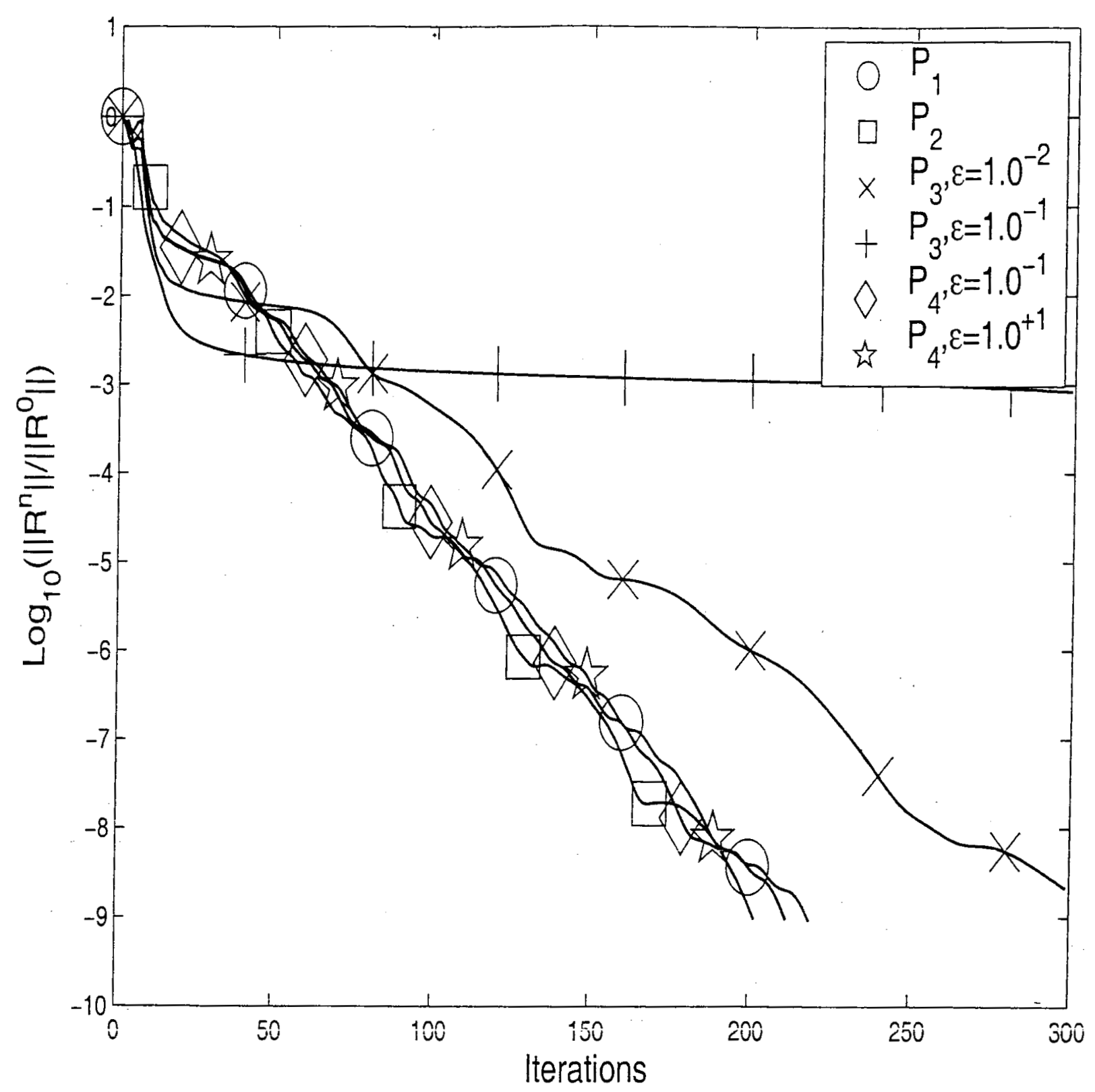

Grid $33 \times 9 \times 9, R e=1000, C F L=1000:$ Plot of normalized $l^{2}$ norm residual $\beta=1$ 


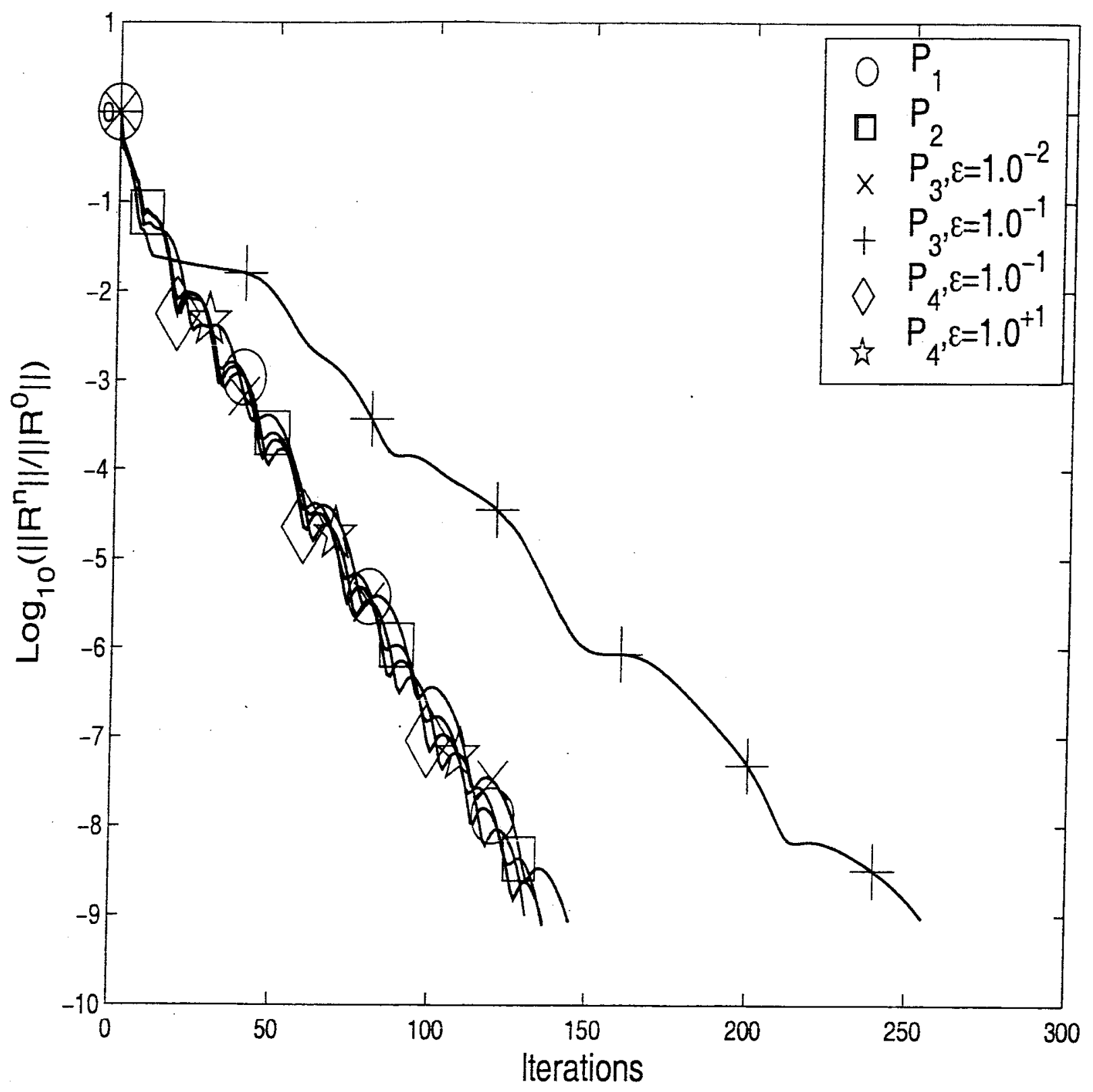

Grid $33 \times 9 \times 9, R e=1000, C F L=1000:$ Plot of normalized $l^{2}$ norm residual $\beta=10$ 


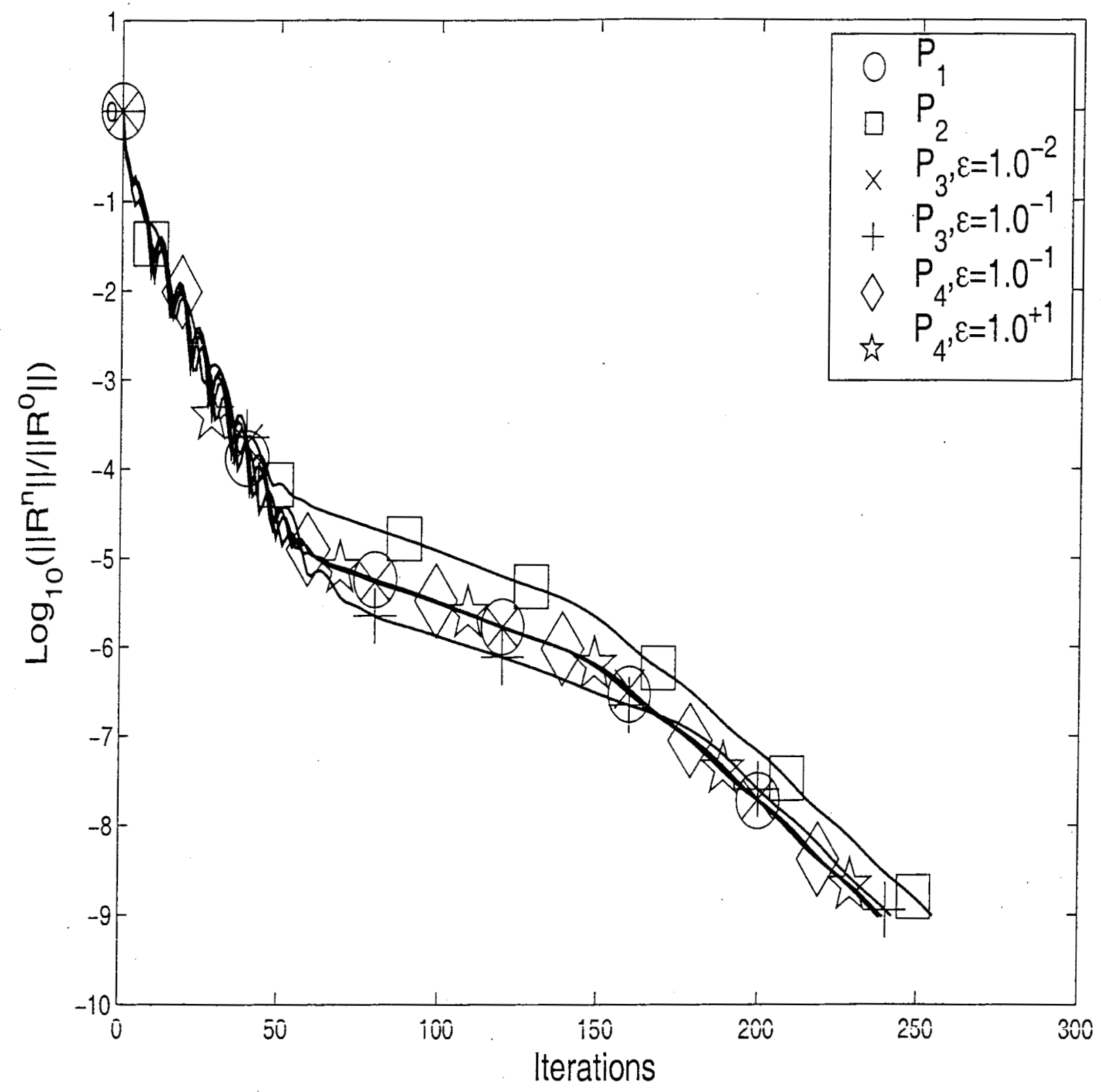

Grid $33 \times 9 \times 9, R e=1000, C F L=1000:$ Plot of normalized $l^{2}$ norm residual $\beta=100$ 


\section{Viscous Circular Pipe}

Viscous flow in a circular pipe of radius one and length ten is computed. A Reynolds number of 1000 is used for which an exact solution is derived. A Grid of dimension $65 \times 33 \times 33$ is used. The table below displays the number of iterations required to converge on the finest mesh. The third and fourth methods fail to converge for low $C F L$ numbers and the artificial dissipation scheme is especially sensitive to the $\epsilon$ parameter. For high $C F L$ and high $\beta$ the differential preconditioning becomes effective, but a moderate $\beta$ must be used for accuracy purposes.

\begin{tabular}{|r|r|l|l|l|l|l|l|l|}
\hline & & $\mathrm{P}=1$ & $\mathrm{P}=2$ & & $\mathrm{P}=3$ & & $\mathrm{P}=4$ & \\
\hline $\mathrm{CFL}$ & $\beta$ & & & $\epsilon=$ & 0.01 & 0.10 & 0.10 & 10.0 \\
\hline 1 & 1 & 352 & 363 & & 518 & $>2500$ & 399 & $>2500$ \\
\hline & 10 & 290 & 301 & & 364 & 500 & 374 & $>2500$ \\
\hline & 100 & 734 & 743 & & 753 & 1044 & 922 & $>2500$ \\
\hline \hline 1000 & 1 & 343 & 355 & & 509 & $>2500$ & 343 & 345 \\
\hline & 10 & 281 & 286 & & 362 & 503 & 281 & 295 \\
\hline & 100 & 744 & 754 & & 748 & 1030 & 743 & 688 \\
\hline
\end{tabular}




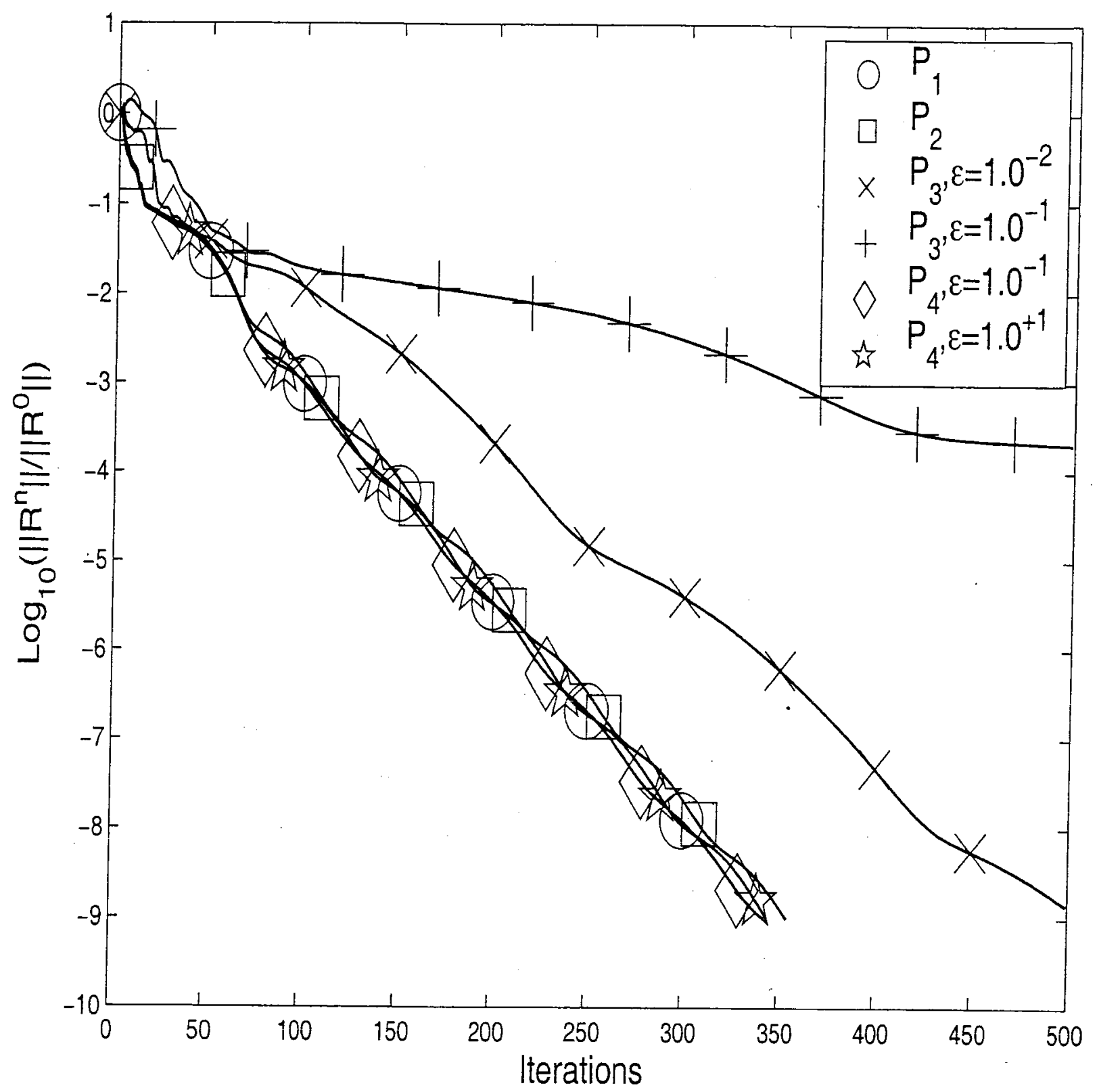

Grid $65 \times 33 \times 33, R e=1000, C F L=1000:$ Plot of normalized $l^{2}$ norm residual $\beta=1$ 


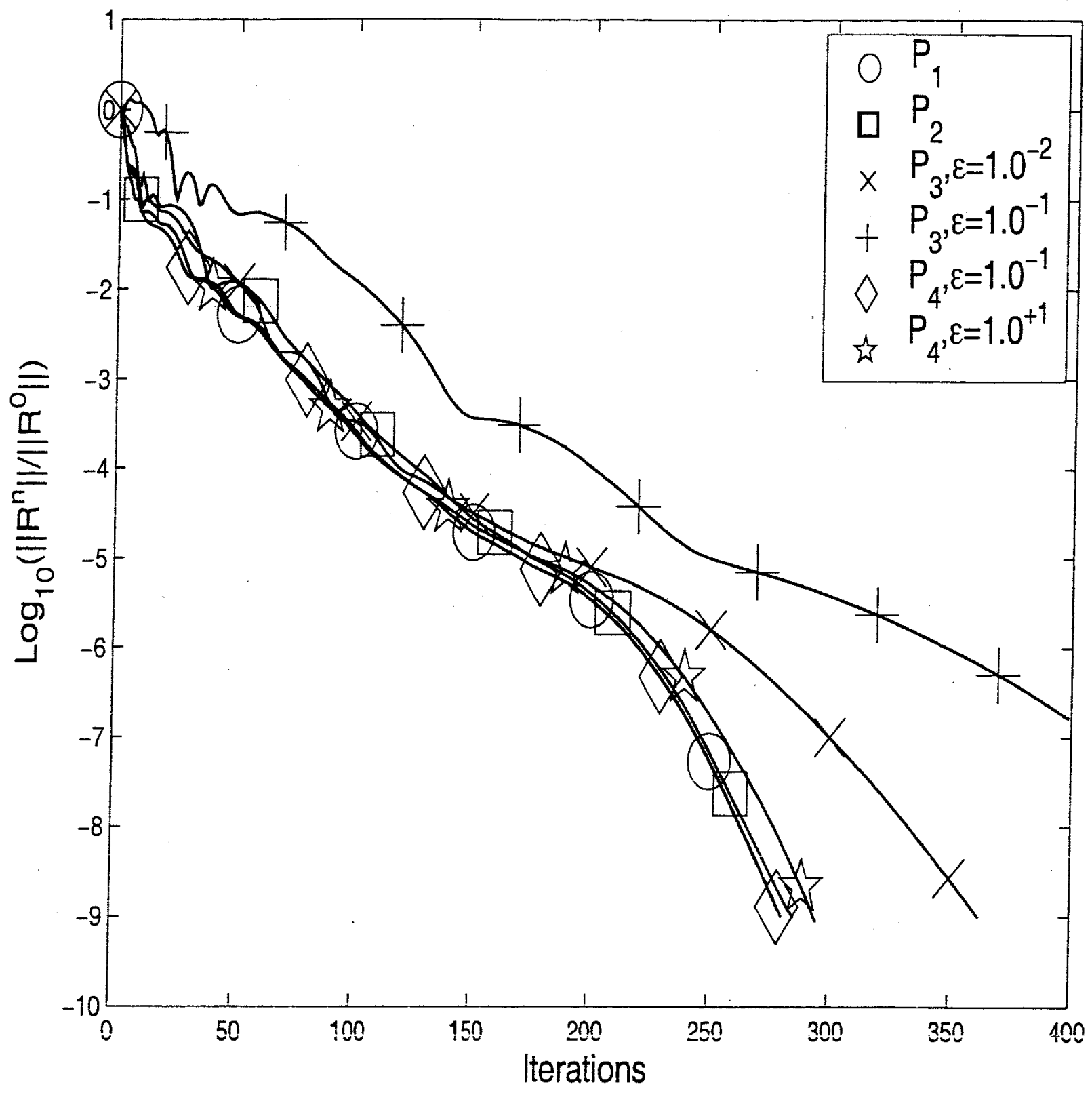

Grid $65 \times 33 \times 33, R e=1000, C F L=1000$ : Plot of normalized $l^{2}$ norm residual $\beta=10$ 


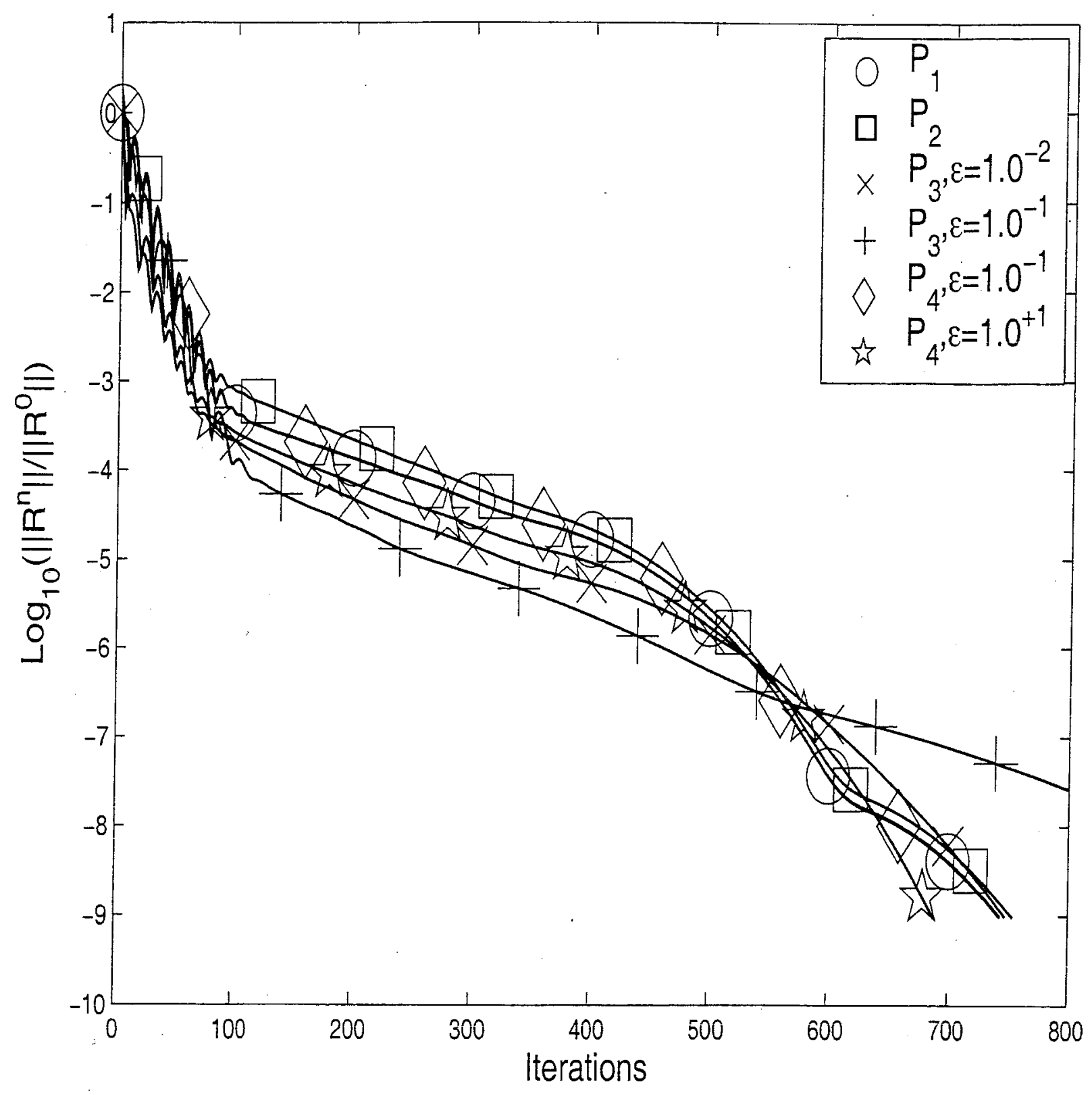

Grid $65 \times 33 \times 33, R e=1000, C F L=1000:$ Plot of normalized $l^{2}$ norm residual $\beta=100$ 


\section{Viscous Square Duct with Strong Curvature}

Viscous flow in a square duct with a $90^{\circ}$ bend is used for the final test. The geometry used is described in Humphrey [5], where experimental results were obtained for Reynolds number 790. A grid of dimension $65 \times 33 \times 33$ is used. The table below displays the number of iterations required to converge. The symbol $* * * *$ denotes the method failed to converge. Robustness is an issue for the third and fourth schemes. Comparing the experimental data with the computed solutions we observe that using $\beta>10$ leads to poor solution accuracy and $\beta=1$ is the most accurate.

\begin{tabular}{|r|r|l|l|l|l|l|l|l|}
\hline CFL & $\beta$ & & & & & & & \\
\hline & $\beta$ & & & 0.01 & 0.10 & $P=4$ & \\
\hline \hline 1 & 1 & 697 & 655 & & 2764 & 5481 & 1141 & $* * * *$ \\
\hline & 10 & 341 & 365 & & $* * * *$ & $* * * *$ & 388 & $* * * *$ \\
\hline & 100 & 524 & 594 & & $* * * *$ & $* * * *$ & $* * * *$ & $* * * *$ \\
\hline \hline 1000 & 1 & 693 & 653 & & 2760 & 5483 & 693 & 750 \\
\hline & 10 & 338 & 361 & & $* * * *$ & $* * * *$ & 338 & 344 \\
\hline & 100 & 534 & $* * * *$ & & $* * * *$ & $* * * *$ & 534 & 543 \\
\hline
\end{tabular}




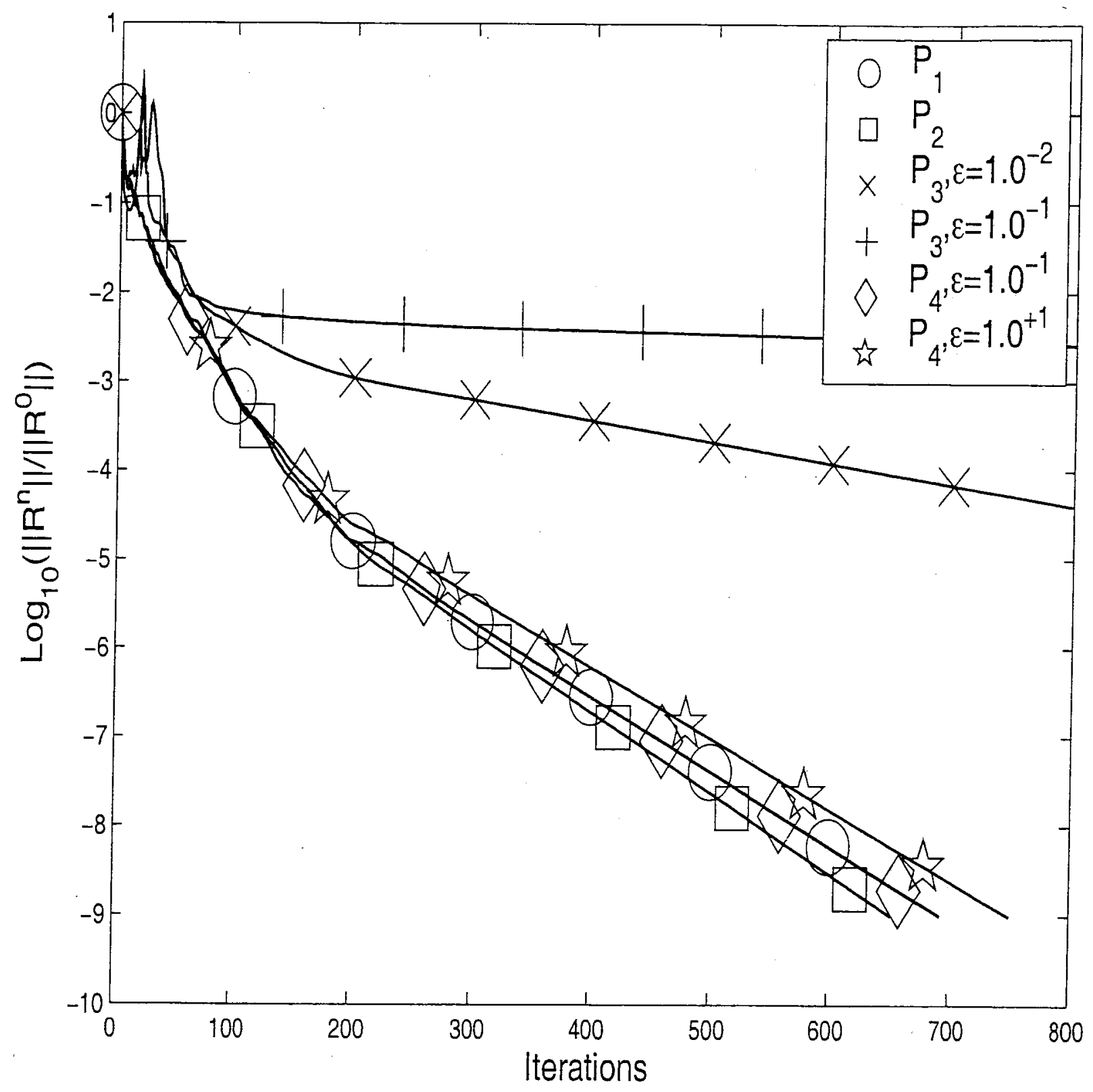

Grid $65 \times 33 \times 33, R e=1000, C F L=1000:$ Plot of normalized $l^{2}$ norm residual $\beta=1$ 


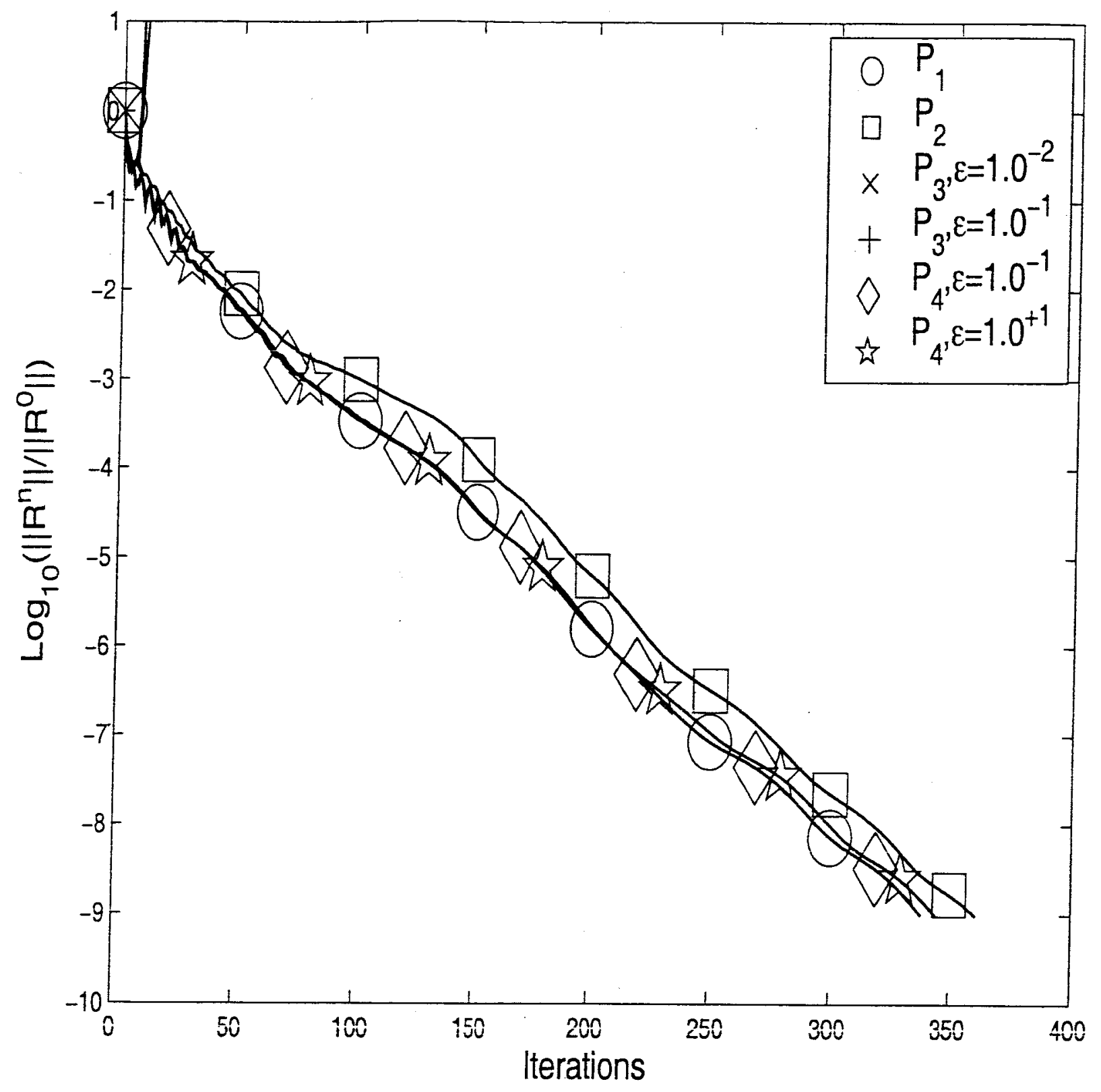

Grid $65 \times 33 \times 33, R e=1000, C F L=1000:$ Plot of normalized $l^{2}$ norm residual $\beta=10$ 


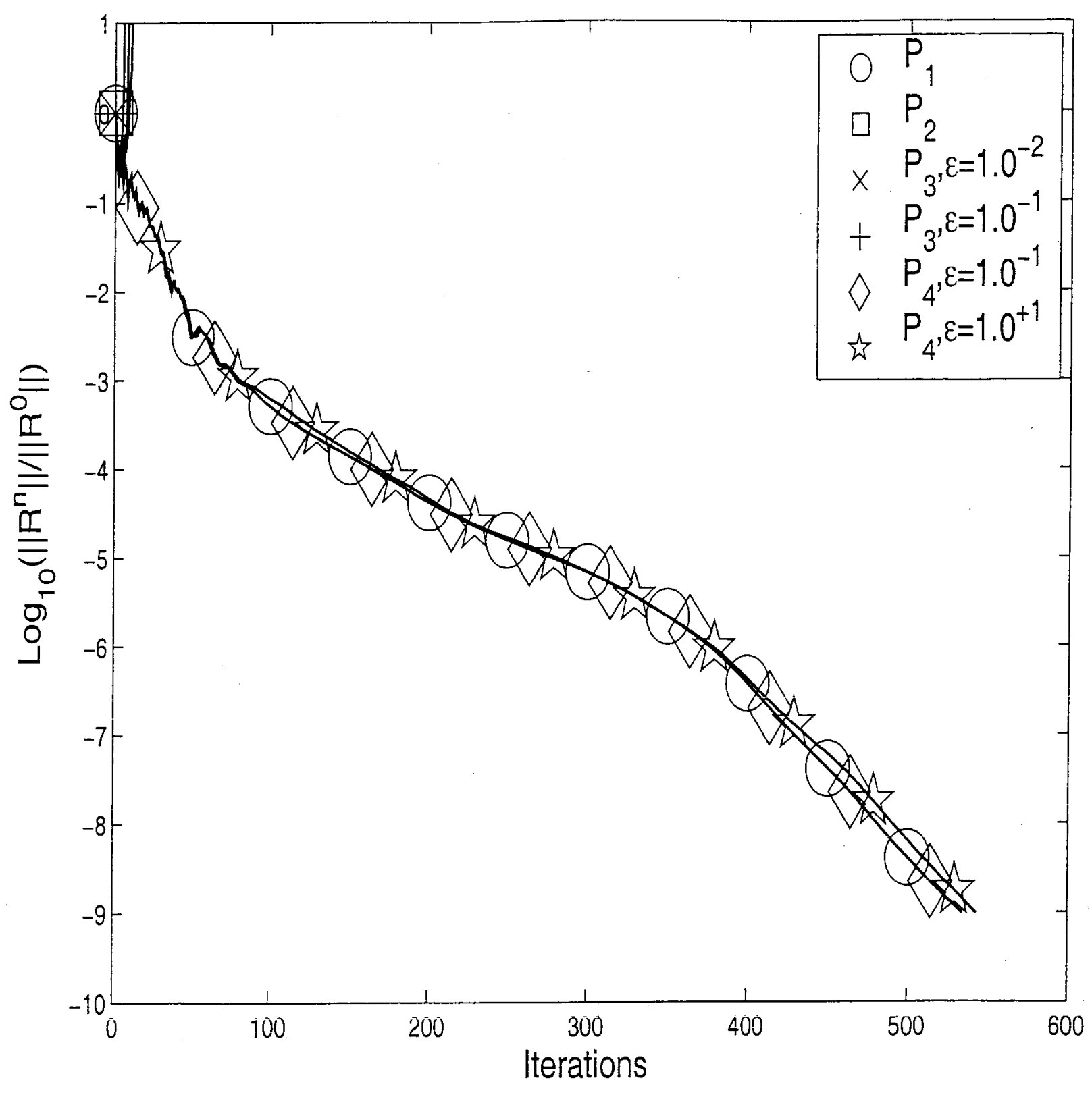

Grid $65 \times 33 \times 33, R e=1000, C F L=1000:$ Plot of normalized $l^{2}$ norm residual $\beta=100$ 


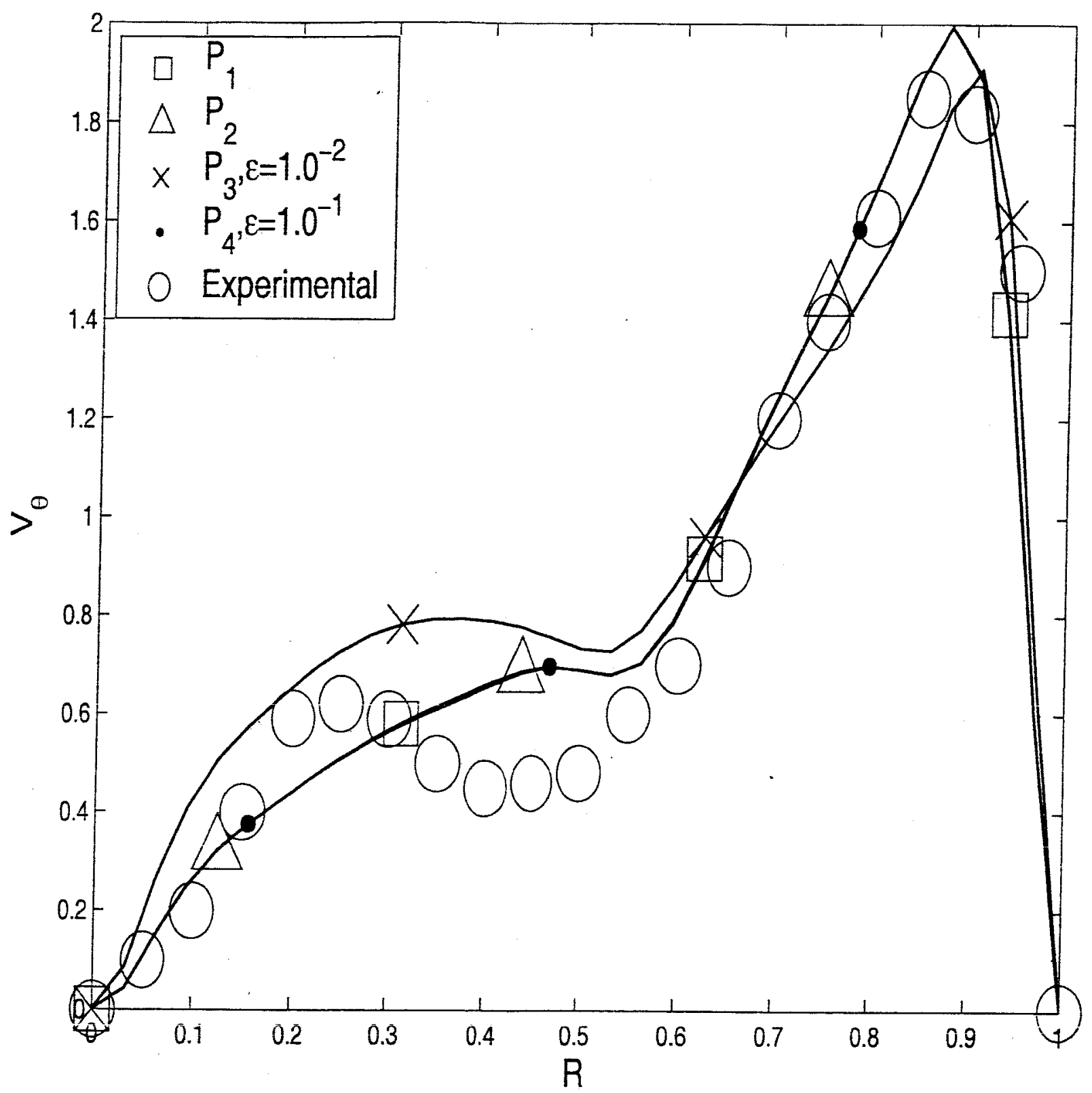

Comparison of the solution at $\theta=90^{\circ}$ and $\beta=1$. 


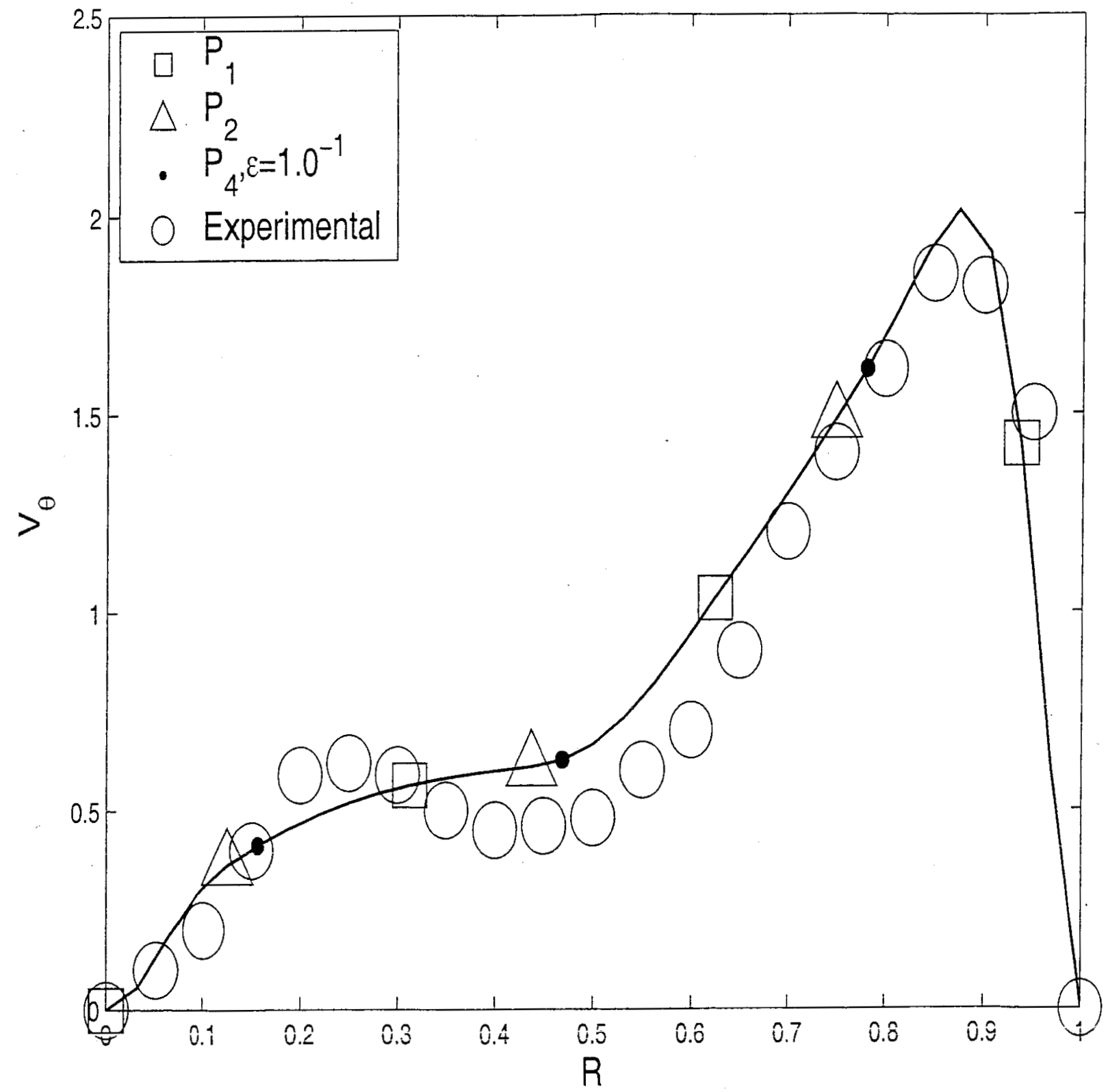

Comparison of the solution at $\theta=90^{\circ}$ and $\beta=10$. 


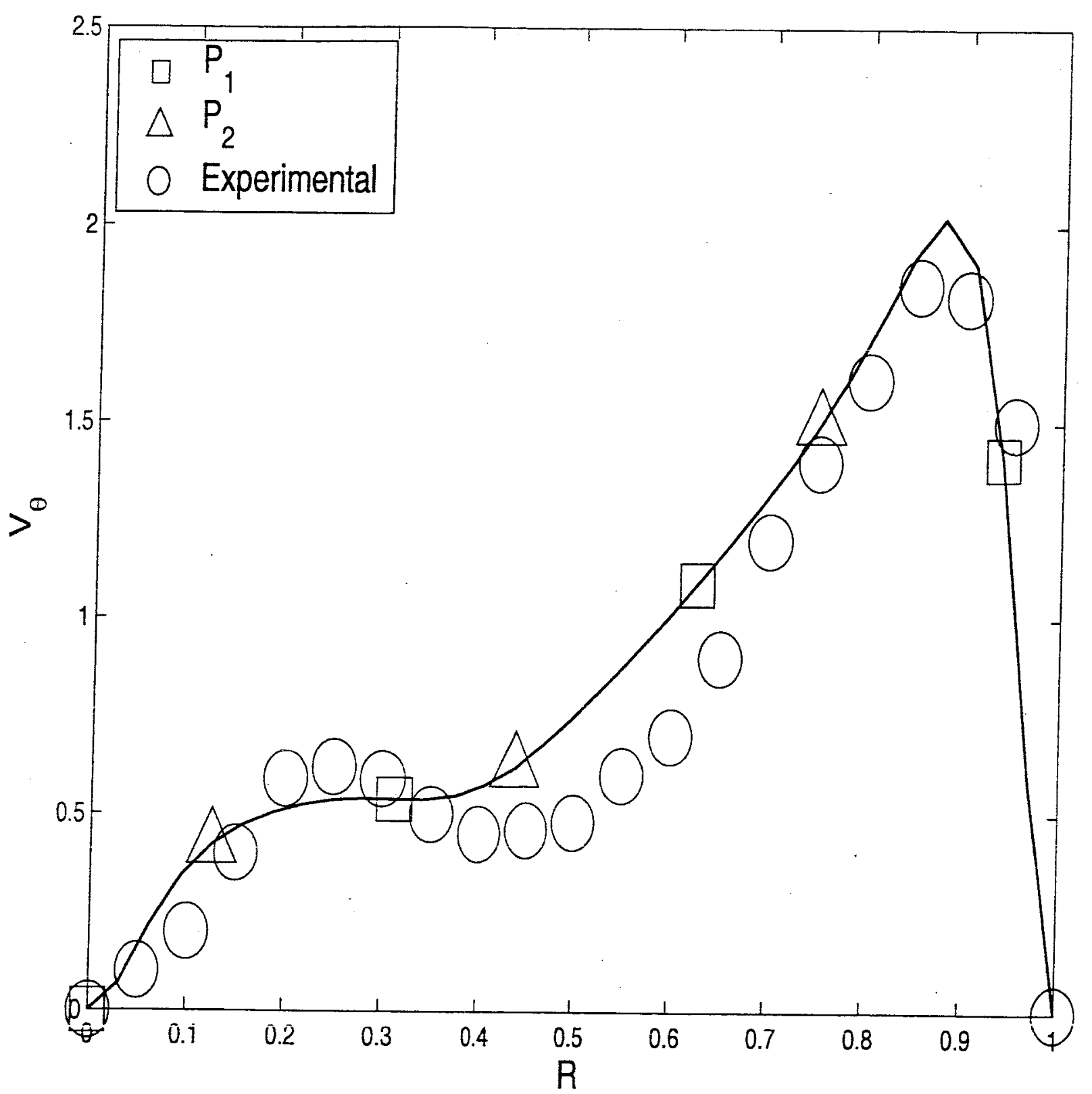

Comparison of the solution at $\theta=90^{\circ}$ and $\beta=100$. 


\section{Conculsion}

Four variations of the artificial compressibility method have been implemented and compared on a series of test problems. The classical and generalized artificial compressibility methods have almost identical convergence rates on each test case for every combination of the $C F L$ and $\beta$ parameters. The artificial dissipation and differential preconditioning methods were not able to converge for all parameters on certain problems showing a lack of robustness for these methods. High values of $\beta$ lead to poor accuracy for all the methods considered. For moderate values of $1 \leq \beta \leq 10$ the classical and generalized versions appear to be the most accurate. These two versions will be evaluated for more complicated engineering applications. 


\section{Bibliography}

1. A.J. Chorin, A Numerical Method for Solving Incompressible Flow Problems, J. Comp. Phys., Vol. 2, 1967

2. C. Kiris, D. Kwak, and S. Rogers, Incompressible Navier-Stokes Solvers in Primitive Variables and Their Applications to Steady and Unsteady Flow, Numerical Simulations of Incompressible Flows, World Scientific Publishing, 2003

3. E. Turkel and R. Radespiel, Preconditioning Methods in Multidimensional Aerodynamics, VKI Lecture Notes, 1997

4. J. Shen, Pseudo-Compressibility Methods for the Unsteady Incompressible Navier-Stokes Equations, Beijing Symposium on Nonlinear Evolution Equations and Infinite Dynamical Systems, 1997

5. J.A. Humphrey, A.M. Taylor, and J. Whitelaw Laminar Flow in a Square Duct of Strong Curvature, J. Fluid Mech., Vol. 83, 1977 